\title{
Effects of the Forsus Fatigue-resistant Device on Skeletal Class II Malocclusion Correction
}

\author{
Hegang $\mathrm{Li}^{1}$, Xun Ren², Yun $\mathrm{Hu}^{3}$, Lijun Tan ${ }^{4}$
}

\begin{abstract}
Aim: To test the hypothesis that skeletal and dentoalveolar effects are both important in skeletal class II malocclusion corrected with the Forsus fatigue-resistant device (FRD).

Materials and methods: A total of 35 patients ( 16 females and 19 males; age $12.0 \pm 0.6$ years) with skeletal class II malocclusion treated with the Forsus FRD were included. Lateral cephalometric radiographies before and after treatment were collected. Cephalometric analysis and superimpositions were applied. Pancherz's analysis was performed to discover the skeletal and dentoalveolar effects on all patients and $60 \%$ contribution was set as a milestone to classify. Statistical comparisons were performed by paired $t$ testing $(p<0.05)$.

Results: The mean treatment period of the Forsus FRD was $6.4 \pm 0.2$ months. All patients (AG) have been corrected to class I molar relationship in three mechanisms: 15 patients in the skeletal group (SG), 10 patients in the dentoalveolar group (DG), and 10 patients in the skeletal and dentoalveolar group (SDG). Four groups showed a significant change in skeletal sagittal relationship improvement $(p<0.05)$. The AG, SG, and SDG showed a significant improvement in the growth of the mandible (Co-Go, Go-Pog, and Co-Gn, $p<0.05)$. The DG showed a significant improvement in the growth of the mandibular body (Go-Pog, $p<0.05$ ).

Conclusion: Three mechanisms were found in skeletal class II malocclusion corrected with the Forsus FRD. Skeletal and dentoalveolar effects are both important in skeletal class II malocclusion corrected with the Forsus FRD. And skeletal and dentoalveolar effects played differential roles in different cases.

Clinical significance: The mechanism of skeletal class II correction with Forsus FRD may divide into mandibular growth, dentoalveolar effects, and both.

Keywords: Class II malocclusion, Dentoalveolar effects, Forsus fatigue-resistant device, Skeletal effects.

The Journal of Contemporary Dental Practice (2020): 10.5005/jp-journals-10024-2748
\end{abstract}

\section{INTRODUCTION}

Skeletal class II malocclusion is a common orthodontic situation. The mechanisms of skeletal class II malocclusion are mandibular retrognathia and (or) maxillary prognathism. Mandibular retrognathia is found to be the primary cause. ${ }^{1}$ Removable or fixed mandibular advancement appliances have been proven to be effective to correct skeletal class II malocclusion before or during the pubertal spurt. ${ }^{2}$ Fixed appliances, such as Herbst, Jasper Jumper, and Forsus fatigue-resistant device (FRD), are popular for their independence of patient cooperation.

Forsus FRD (3M Unitek, Monrovia, California) was developed by Bill Vogt in 2001. ${ }^{3}$ Forsus FRD allows flexibility in the position of the mandible as compared to the Herbst appliance, which advances the mandible rigidly. ${ }^{2}$ Forsus FRD is a fairly effective fixed functional appliance. It can exert consistent force, the level of which can be adjusted by choosing the pushrod in five different sizes to satisfy varies clinical situation. ${ }^{4}$ A $226 \mathrm{~g}$ force can be delivered when the spring of the Forsus FRD is compressed to $12 \mathrm{~mm} .{ }^{4}$ Class II correction with Forsus FRD can be achieved in 4-6 months, and stable mandibular advancement can be achieved in the following 24 months after the 6 months' active treatment. ${ }^{5}$

The effects of fixed appliances for class II correction are controversial in the extent of skeletal or dentoalveolar effects. ${ }^{6}$ Articles available on the Forsus FRD are mainly focused on comparing the skeletal and dentoalveolar effects with other appliances. ${ }^{7}$ Few articles were published to reveal the differential effects of the Forsus FRD. The aim of the present study was to reveal the differential effects of the Forsus FRD on the skeletal (mandibule)
${ }^{1,4}$ Department of Orthodontics, State Key Laboratory of Oral Diseases, National Clinical Research Center for Oral Disease, West China Hospital of Somatology, Sichuan University, Chengdu, Sichuan, China

${ }^{2}$ Department of Stomatology, Sichuan Academy of Medical Sciences, Sichuan Provincial People's Hospital, Chengdu, Sichuan, China

${ }^{3}$ Department of Preventive Dentistry, State Key Laboratory of Oral Diseases, National Clinical Research Center for Oral Disease, West China Hospital of Somatology, Sichuan University, Chengdu, Sichuan, China

Corresponding Author: Lijun Tan, Department of Orthodontics, State Key Laboratory of Oral Diseases, National Clinical Research Center for Oral Disease, West China Hospital of Somatology, Sichuan University, Chengdu, Sichuan, China, Phone:+86 13458515333, e-mail:orthotan@ vip.163.com

How to cite this article: $\mathrm{Li} \mathrm{H}$, Ren $\mathrm{X}, \mathrm{Hu} \mathrm{Y}$, et al. Effects of the Forsus Fatigue-resistant Device on Skeletal Class II Malocclusion Correction. J Contemp Dent Pract 2020;21(1):105-112.

Source of support: Nil

Conflict of interest: None

and dentoalveolar effect in skeletal class II patients. The hypothesis is that both skeletal and dentoalveolar effects play important roles in skeletal class II malocclusion correction with the Forsus FRD.

\section{Materials and Methods}

A total of 35 subjects (age $12.0 \pm 0.6$ years) with skeletal class II malocclusions (16 females and 19 males) were included in the 
present study. Subjects were obtained from the Department of Orthodontics, West China Hospital of Stomatology, Sichuan University.

The inclusion criteria for present study are as follows: (1) skeletal class II malocclusion in the early permanent dentition, (2) a retrognathic mandible, (3) in or before the pubertal spurt (evaluated by cephalometric radiographs, stages 2 and 3 based on the method of an improved version of the cervical vertebral maturation method), ${ }^{8}$ (4) the MP-SN angle not exceeding $40^{\circ}$ and the MP-FH angle not exceeding $33^{\circ}$, and (5) no signs of temporomandibular joint disease. The exclusion criteria include: (1) class II malocclusion due to a prognathic maxilla, (2) patients in the postpubertal period, (3) vertical growth pattern, (4) poor oral hygiene, and (5) temporomandibular joint disease.

Bands with a headgear tube and $0.022 \times 0.028$ inches slot preadjusted edgewise brackets were ligated with $0.019 \times 0.025$ inches stainless steel archwires in all patients. The entire upper and lower arches were laced tightly under the archwires. The wires were cinched back in both arches before the insertion of the Forsus FRD. The EX2 module of the Forsus FRD was selected and fixed according to the manufacturer's instruction and removed after mean time $6.4 \pm 0.2$ months

The effects of Forsus FRD were evaluated by lateral cephalometric radiographs before treatment (T1) and after treatment (T2). All the cephalometric radiographs were taken on the same X-ray equipment. All the cephalometric tracing, analysis, and superposition were done with Dolphin Imaging Software Version 11.7 (Dolphin Imaging and Management Solutions, Chatsworth, California). The skeletal and dentoalveolar contributions to skeletal class II correction were analyzed according to the method of Pancherz. ${ }^{9}$ Sixty percent was set as a milestone to classify differential contributions of skeletal and dentoalveolar effects. All measurements were evaluated three times, repeated after a 2-week interval by the same orthodontist (HG.L) and checked by another orthodontist (Y.H). Any disagreements were resolved to the satisfaction of both observers. Changes within each group between after (T2) and before (T1) measurements in skeletal, dentoalveolar, and soft tissue were evaluated by paired $t$ testing with Statistical Package for Social Sciences (SPSS; Version 18.0; IBM, Armonk, NY). The $5 \%$ significance level was set to interpret all statistical tests in the present study.

\section{Results}

Class I or overcorrected class I canine and molar relationship was achieved for all patients after $6.4 \pm 0.2$ months. Skeletal, dentoalveolar, and soft tissue changes between after (T2) and before (T1) treatment are shown in Table 1. Pancherz's analysis is shown in Table 2 and Figure 1. Cephalometric superimpositions are shown in Figure 2. Groups divided by Pancherz's analysis are shown in Table 3.

Measurements in all patients (all patients group, AG) were shown as follows. Significant improvement in the skeletal sagittal relationship was detected (ANB: $-1.3 \pm 1.1^{\circ}$ and Wits: $-3.4 \pm 2.6 \mathrm{~mm} ; p<0.05$ ). Significant clockwise rotation was detected in the functional occlusal plane (OP-SN: $\left.3.9 \pm 3.2^{\circ} ; p<0.05\right)$. The maxillary incisors retroclined significantly (U1-NA: $-4.6 \pm 5.3^{\circ}$ and U1-NA: $-1.2 \pm 1.8 \mathrm{~mm} ; p<0.05$ ) and extruded significantly (U1-PP: $1.6 \pm 1.7 \mathrm{~mm} ; p<0.05)$. The mandibular incisors proclined significantly (L1-NB: $10.2 \pm 5.7^{\circ}$ and L1-NB: $2.8 \pm 2.1 \mathrm{~mm} ; p<0.05)$. The mandibular molars moved mesially and extruded significantly (L6-MP: $2.6 \pm 1.7 \mathrm{~mm}$ and L6-PTv: $5.1 \pm 3.0 \mathrm{~mm} ; p<0.05)$. The mandible exhibited significant growth (Co-Go: $3.0 \pm 3.1 \mathrm{~mm}$, Go-Pog: $2.2 \pm 2.4 \mathrm{~mm}$, and Co-Gn: $4.0 \pm 4.0 \mathrm{~mm} ; p<0.05)$. Soft tissue measurements revealed a significant retrusion of the upper lip and protrusion of the lower lip to the E-plane $(-1.2 \pm 1.6 \mathrm{~mm}$ and $1.0 \pm 1.9 \mathrm{~mm}$, respectively; $p<0.05)$.

Skeletal and dentoalveolar contributions varied in different cases (Table 2 and Fig. 1). Three kinds of mechanisms were contributed to the correction of class II malocclusion. Correction of 15 patients came mainly from mandibular growth (more than $60 \%$ skeletal contribution) (skeletal group, SG). Correction of 10 patients came mainly from the mesial movement of mandibular dentoalveolar change (more than $60 \%$ dentoalveolar contribution) (dentoalveolar group, DG). And correction of 10 patients came from both mandibular growth and mandibular molar mesial movement (skeletal and dentoalveolar group, SDG) (Fig. 2).

The cephalometric measurements of skeletal, dentoalveolar, and soft tissue changes in SG, DG, and SDG are shown in Table 3. All the following significant changes could be detected in all three groups: correction in skeletal sagittal relationship according to ANB and Wits appraisal $\left(-1.4 \pm 1.2^{\circ}\right.$ and $-4.0 \pm 2.8 \mathrm{~mm},-1.3 \pm 1.0^{\circ}$ and $-2.4 \pm 2.0 \mathrm{~mm},-1.2 \pm 1.2^{\circ}$ and $-3.5 \pm 2.7 \mathrm{~mm}$, respectively; $p<0.05)$; clockwise rotation of the functional occlusal plane according to OP-SN $\left(4.3 \pm 2.7^{\circ}, 2.9 \pm 2.9^{\circ}, 4.2 \pm 4.2^{\circ}\right.$, respectively; $p<0.05)$; the maxillary incisors retroclined and extruded according to U1-NA $\left(-4.7 \pm 6.7^{\circ},-3.5 \pm 4.2^{\circ},-5.3 \pm 3.9^{\circ}\right.$, respectively; $\left.p<0.05\right)$ and U1-PP $(1.8 \pm 1.7 \mathrm{~mm}, 1.3 \pm 1.8 \mathrm{~mm}, 1.6 \pm 1.7 \mathrm{~mm}$, respectively; $p<0.05)$; the mandibular incisors proclined according to L1-NB $\left({ }^{\circ}\right)$ and L1-NB $(\mathrm{mm})\left(9.6 \pm 4.1^{\circ}\right.$ and $2.8 \pm 2.3 \mathrm{~mm}, 12.6 \pm 8.0^{\circ}$ and $3.3 \pm 2.5 \mathrm{~mm}, 8.6 \pm 4.9^{\circ}$ and $2.2 \pm 1.4 \mathrm{~mm}$, respectively; $p<0.05$ ); the mandibular molars moved mesially and extruded according to L6-MP and L6-PTv $(2.8 \pm 1.9$ and $4.3 \pm 2.2 \mathrm{~mm}, 2.5 \pm 1.5$ and $5.2 \pm 3.4 \mathrm{~mm}, 2.6 \pm 1.8$ and $5.6 \pm 2.5 \mathrm{~mm}$, respectively; $p<0.05)$.

Significant improvement in the growth of the mandible in all dimension was detected in SG and SDG (Co-Go: $4.1 \pm 3.3$ and $2.3 \pm 2.3 \mathrm{~mm}$, Go-Pog: $2.2 \pm 2.2$ and $2.6 \pm 3.0 \mathrm{~mm}$, Co-Gn: $4.9 \pm$ 3.3 and $4.0 \pm 4.6 \mathrm{~mm}$, respectively; $p<0.05)$. Significant growth of the mandibular body could be detected in DG (Go-Pog: $1.8 \pm$ $2.3 \mathrm{~mm}, p<0.05$ ). Significant maxilla restriction (SNA: $-0.4 \pm 0.7$, $p<0.05)$ and anti-clockwise rotation in the mandibular plane (MP-FH: $-1.1 \pm 2.1, p<0.05$ ) could be revealed in SG only.

\section{Discussion}

This study was trying to discover the comprehensive effects of the Forsus FRD on skeletal class II malocclusion correction. Pancherz's analysis combined with cephalometric superimposition were able to reveal the sagittal skeletal and dentoalveolar contribution to the correction. No consensus ${ }^{5}$ was reached in the skeletal effects of the Forsus FRD until now. Some researches ${ }^{5,10}$ have revealed that the Forsus FRD was effective in skeletal class II correction mainly by combination of maxilla growth restriction and mesial movement of mandibular incisors and first molars. It was reported that the Forsus FRD brought no statistically significant skeletal changes in some studies. ${ }^{11}$ Differential response was detected in the present study. No previous paper was focused on differential contributions of skeletal and dentoalveolar effects in class II correction with the Forsus FRD. In the present study, the proportion of skeletal effects varied from 92.9 to $2 \%$ in overjet correction and 86.2 to $2 \%$ in molar relationship correction. Correction of class II malocclusion 
Table 1: Cephalometric measurements in all patients

\begin{tabular}{|c|c|c|c|c|c|}
\hline & $T 1($ mean $\pm S D)$ & $T 2($ mean $\pm S D)$ & $T 2-T 1($ mean $\pm S D)$ & $t$ & $p$ value \\
\hline \multicolumn{6}{|l|}{ Skeletal sagittal } \\
\hline SNA $\left(^{\circ}\right)$ & $81.5 \pm 3.6$ & $81.2 \pm 3.6$ & $-0.3 \pm 0.9$ & 1.98 & 0.06 \\
\hline SNB $\left(^{\circ}\right)$ & $75.4 \pm 3.4$ & $76.4 \pm 3.5$ & $1.0 \pm 1.2$ & -4.99 & $0.00^{*}$ \\
\hline ANB $\left(^{\circ}\right)$ & $6.0 \pm 1.9$ & $4.7 \pm 1.8$ & $-1.3 \pm 1.1$ & 7.17 & $0.00^{*}$ \\
\hline Wits (mm) & $3.8 \pm 3.2$ & $0.4 \pm 2.5$ & $-3.4 \pm 2.6$ & 7.78 & $0.00^{*}$ \\
\hline \multicolumn{6}{|l|}{ Skeletal vertical } \\
\hline GoGn-FH $\left(^{\circ}\right)$ & $25.1 \pm 6.1$ & $25.0 \pm 6.5$ & $-0.1 \pm 2.2$ & 0.35 & 0.73 \\
\hline GoGn-PP $\left({ }^{\circ}\right)$ & $23.7 \pm 5.7$ & $23.6 \pm 5.5$ & $-0.1 \pm 2.2$ & 0.23 & 0.82 \\
\hline GoGn-SN $\left(^{\circ}\right)$ & $33.3 \pm 6.3$ & $33.1 \pm 6.8$ & $-0.3 \pm 2.1$ & 0.70 & 0.49 \\
\hline OP-SN $\left(^{\circ}\right)$ & $18.0 \pm 4.1$ & $21.9 \pm 4.8$ & $3.9 \pm 3.2$ & -7.19 & $0.00^{*}$ \\
\hline Y-axis $\left(^{\circ}\right)$ & $67.0 \pm 5.8$ & $67.2 \pm 5.4$ & $0.2 \pm 1.7$ & -0.70 & 0.49 \\
\hline $\mathrm{N}-\mathrm{Me}(\mathrm{mm})$ & $109.7 \pm 6.9$ & $113.5 \pm 7.1$ & $3.8 \pm 4.9$ & -4.66 & $0.00^{*}$ \\
\hline (S-Go/N-Me) (\%) & $67.5 \pm 5.3$ & $67.5 \pm 6.4$ & $0.0 \pm 2.9$ & -0.01 & 0.99 \\
\hline (ANS-Me/N-Me) (\%) & $52.5 \pm 1.5$ & $53.0 \pm 1.5$ & $0.5 \pm 1.2$ & -2.39 & $0.02^{*}$ \\
\hline \multicolumn{6}{|l|}{ Mandible } \\
\hline Co-Gn (mm) & $103.2 \pm 6.5$ & $107.2 \pm 7.3$ & $4.0 \pm 4.0$ & -6.00 & $0.00^{*}$ \\
\hline Co-Go (mm) & $54.0 \pm 6.1$ & $57.0 \pm 7.1$ & $3.0 \pm 3.1$ & -5.80 & $0.00^{*}$ \\
\hline Go-Pog (mm) & $62.8 \pm 5.0$ & $65.0 \pm 5.3$ & $2.2 \pm 2.4$ & -5.39 & $0.00^{*}$ \\
\hline \multicolumn{6}{|l|}{ Dentoalveolar } \\
\hline U1-L1 ( $\left.{ }^{\circ}\right)$ & $119.9 \pm 13.6$ & $114.2 \pm 9.2$ & $-5.7 \pm 11.7$ & 2.90 & $0.01 *$ \\
\hline \multicolumn{6}{|l|}{ Upper } \\
\hline U1-NA $\left(^{\circ}\right)$ & $28.1 \pm 7.2$ & $23.5 \pm 5.8$ & $-4.6 \pm 5.3$ & 5.11 & $0.00^{*}$ \\
\hline U1-NA (mm) & $5.6 \pm 2.6$ & $4.4 \pm 2.1$ & $-1.2 \pm 1.8$ & 4.00 & $0.00^{*}$ \\
\hline U1-SN $\left(^{\circ}\right)$ & $108.9 \pm 7.6$ & $104.7 \pm 6.8$ & $-4.2 \pm 5.7$ & 4.30 & $0.00^{*}$ \\
\hline U1-PP (mm) & $26.2 \pm 2.5$ & $27.8 \pm 2.3$ & $1.6 \pm 1.7$ & -5.61 & $0.00^{*}$ \\
\hline U6-PP (mm) & $19.4 \pm 2.4$ & $19.3 \pm 2.5$ & $-0.1 \pm 1.6$ & 0.20 & 0.84 \\
\hline U6-PTV (mm) & $21.8 \pm 3.2$ & $21.6 \pm 3.2$ & $-0.2 \pm 2.7$ & 0.43 & 0.67 \\
\hline \multicolumn{6}{|l|}{ Lower } \\
\hline L1-NB $\left(^{\circ}\right)$ & $27.6 \pm 6.7$ & $37.7 \pm 6.3$ & $10.2 \pm 5.7$ & -10.49 & $0.00^{*}$ \\
\hline L1-NB (mm) & $5.5 \pm 2.2$ & $8.2 \pm 2.4$ & $2.8 \pm 2.1$ & -7.69 & $0.00^{*}$ \\
\hline L1-MP $\left(^{\circ}\right)$ & $98.6 \pm 7.8$ & $108.5 \pm 6.2$ & $9.8 \pm 5.9$ & -9.90 & $0.00^{*}$ \\
\hline L1-MP (mm) & $38.0 \pm 3.3$ & $37.5 \pm 3.5$ & $-0.5 \pm 2.8$ & 1.00 & 0.32 \\
\hline L6-MP (mm) & $28.2 \pm 2.4$ & $30.8 \pm 2.9$ & $2.6 \pm 1.7$ & -9.20 & $0.00^{*}$ \\
\hline L6-PTV (mm) & $19.5 \pm 3.3$ & $24.6 \pm 3.3$ & $5.1 \pm 3.0$ & -9.84 & $0.00 *$ \\
\hline \multicolumn{6}{|l|}{ Soft tissue } \\
\hline ST N-subnasale-ST Pog $\left({ }^{\circ}\right)$ & $160.2 \pm 6.2$ & $161.6 \pm 6.4$ & $1.4 \pm 3.5$ & -2.32 & $0.03^{*}$ \\
\hline ST N-Tip Nose-ST Pog $\left(^{\circ}\right)$ & $139.1 \pm 8.1$ & $138.3 \pm 9.2$ & $-0.8 \pm 4.7$ & 0.98 & 0.33 \\
\hline Upper Lip-E (mm) & $2.3 \pm 1.9$ & $1.1 \pm 1.9$ & $-1.2 \pm 1.6$ & 4.36 & $0.00^{*}$ \\
\hline Lower Lip-E (mm) & $2.3 \pm 2.6$ & $3.3 \pm 2.4$ & $1.0 \pm 1.9$ & -3.12 & $0.00^{*}$ \\
\hline Z angle $\left(^{\circ}\right)$ & $62.4 \pm 7.8$ & $65.1 \pm 7.9$ & $2.7 \pm 5.6$ & -2.90 & $0.01 *$ \\
\hline Upper-lip (mm) & $12.2 \pm 2.3$ & $14.2 \pm 2.6$ & $1.9 \pm 1.7$ & -6.72 & $0.00^{*}$ \\
\hline Total-chin $(\mathrm{mm})$ & $13.8 \pm 2.1$ & $14.7 \pm 2.1$ & $0.9 \pm 1.7$ & -3.17 & $0.00^{*}$ \\
\hline
\end{tabular}

${ }^{*} p \leq 0.05$ (significant)

was resulted from differential combinations of skeletal and dental effects. Therefore, the mechanism for skeletal class II malocclusion correction indeed varied with different cases. It is difficult to simply compare the skeletal and dentoalveolar contributions in the effects of Forsus FRD in different cases with different mechanisms. This can solve the existed controversy to some extent. Different investigated subject may lead to different results. Previous studies analyzed subjects as a whole group and thus missed some information.

The ANB angle and Wits values decreased significantly in four groups. This may result from the skeletal effect of Go-Pog increase in all four groups and maxilla restriction in SG. It is still difficult to distinguish the effects from growth and that of the Forsus FRD. 
Table 2: Pancherz's cephalometric analysis in all patients

\begin{tabular}{|c|c|c|c|c|}
\hline \multirow[b]{2}{*}{ Number } & \multicolumn{2}{|c|}{ Overjet contributing } & \multicolumn{2}{|c|}{ Molar relation contributing } \\
\hline & Skeletal (\%) & $\begin{array}{l}\text { Dentoalveolar } \\
\text { (\%) }\end{array}$ & Skeletal (\%) & $\begin{array}{l}\text { Dentoalveolar } \\
\text { (\%) }\end{array}$ \\
\hline 1 & 60.9 & 39.1 & 59.6 & 40.4 \\
\hline 2 & 60.5 & 39.5 & 68.4 & 31.6 \\
\hline 3 & 63.0 & 37.0 & 77.3 & 22.7 \\
\hline 4 & 60.9 & 39.1 & 65.1 & 34.9 \\
\hline 5 & 77.0 & 23.0 & 71.4 & 28.6 \\
\hline 6 & 80.0 & 20.0 & 75.7 & 24.3 \\
\hline 7 & 76.9 & 23.1 & 68.2 & 31.8 \\
\hline 8 & 71.8 & 28.2 & 70.0 & 30.0 \\
\hline 9 & 70.2 & 29.8 & 66.0 & 34.0 \\
\hline 10 & 79.4 & 20.6 & 78.1 & 21.9 \\
\hline 11 & 85.7 & 14.3 & 85.7 & 14.3 \\
\hline 12 & 61.2 & 38.8 & 73.2 & 26.8 \\
\hline 13 & 92.9 & 7.1 & 82.5 & 17.5 \\
\hline 14 & 90.0 & 10.0 & 83.3 & 16.7 \\
\hline 15 & 89.3 & 10.7 & 86.2 & 13.8 \\
\hline 16 & 36.6 & 63.4 & 32.6 & 67.4 \\
\hline 17 & 37.2 & 62.8 & 34.0 & 66.0 \\
\hline 18 & 39.5 & 60.5 & 38.5 & 61.5 \\
\hline 19 & 35.6 & 64.4 & 36.2 & 63.8 \\
\hline 20 & 39.0 & 61.0 & 39.0 & 61.0 \\
\hline 21 & 13.6 & 86.4 & 12.0 & 88.0 \\
\hline 22 & 33.3 & 66.7 & 29.9 & 70.1 \\
\hline 23 & 2.0 & 98.0 & 2.0 & 98.0 \\
\hline 24 & 5.6 & 94.4 & 3.9 & 96.1 \\
\hline 25 & 20.8 & 79.2 & 20.6 & 79.4 \\
\hline 26 & 53.3 & 46.7 & 57.1 & 42.9 \\
\hline 27 & 47.0 & 53.0 & 47.0 & 53.0 \\
\hline 28 & 49.0 & 51.0 & 43.6 & 56.4 \\
\hline 29 & 50.8 & 49.2 & 45.7 & 54.3 \\
\hline 30 & 48.3 & 51.7 & 48.3 & 51.7 \\
\hline 31 & 51.2 & 48.8 & 55.0 & 45.0 \\
\hline 32 & 59.0 & 41.0 & 60.0 & 40.0 \\
\hline 33 & 58.2 & 41.8 & 45.7 & 54.3 \\
\hline 34 & 54.0 & 46.0 & 50.9 & 49.1 \\
\hline 35 & 42.2 & 57.8 & 47.4 & 52.6 \\
\hline
\end{tabular}

Dentoalveolar changes can be detected in four groups. Significant correction of overjet and molar relationship was related to retroclination of upper incisors and more significant proclination of lower incisors in the present study. Mandibular molars moved mesially and extruded in four groups. Distal movements of maxillary molars have been reported after application of the Forsus $\mathrm{FRD}^{3}$ while similar results can't be detected in the present study.

The reason for different mechanisms in skeletal class II correction after Forsus FRD was unclear. Variations may be due to different inclusive criteria, study methodologies, and difference in anatomy. ${ }^{6,12}$ Facial growth pattern and the gonial angle might affect the extent of skeletal effects. ${ }^{1,9,12}$ Oztoprak et al. ${ }^{13}$ found that no significant skeletal changes can be detected and skeletal class II correction improved primarily from the dentoalveolar effect when the study sample was after the pubertal spurt. Certainly, it is difficult to exclude the influence of growth from the skeletal effect in the present study, especially that in the present study four groups showed mandibular body growth. And this is a long-lasting debate in functional appliances. It can be summarized that Forsus FRD can result in mesial dentoalveolar movement definitely and may induce mandibular growth. And the contributions of skeletal and dentoalveolar effects varied in different cases. It is hard to conclude the effects of the Forsus FRD in one pattern. Another possible reason for the different mechanism is the masticatory muscle force. Heavy masticatory force tends to move the mandible back. Force generated by the Forsus FRD move the arch in opposite direction and resulted in more dentoalveolar effects.

The clockwise rotation of the functional occlusal plane was detected in all patients in the present study. This was mainly resulted from extrusion of mandibular molars and retroclination of maxillary incisors. These findings are similar to the previous studies with fixed functional appliances. ${ }^{3,14}$ At the same time, the anticlockwise rotation in the mandibular plane was observed in SG only. The reason for this might be that mandibular ramus growth allowed anticlockwise rotation of the mandibular plane. ${ }^{15}$

The effect of the Forsus FRD on the temporomandibular joint (TMJ) is the most concerned subject. The studies on this project were controversial. ${ }^{13,16,17}$ It has been revealed that functional appliances have no adverse effects on TMJ at various ages from puberty to adulthood. ${ }^{16}$ Forsus FRD displaces the mandible forward, translates the condyle out of the condylar fossa, and transmits forces to the dentition and the basal bone. In the present study, values related to the mandible (Co-Gn, Co-Go, and Go-Pog) increased significantly in AG, SG, and SDG patients. Temporomandibular joint modification can be one of the explanations for these changes. It has been revealed by previous studies., ${ }^{5,16,18}$ Karacay et al. ${ }^{18}$ found the adaptive growth in the condyle after a 5.2 months treatment period with Forsus FRD. Franchi ${ }^{5}$ found that the Forsus FRD induced a $1.5 \mathrm{~mm}$ increase in the mandibular ramus height (Co-Go) compared with untreated patients. Sex difference was not included in the present paper. Gomes and Lima ${ }^{17}$ found that there was no clear conclusion about the effects of sex differences on annual mandibular growth rates in adolescents. Another possible explanation for the mandibular increase might be the change in the pogonion location or the repositioning of the anterior mandible rather than its forward growth. ${ }^{13}$ Values related to the length of the mandibular body (Go-Pog) increased significantly in DG patients. It could be the contribution of the differential growth of the mandible.

Lower incisors proclined significantly in four groups. This rang a bell on the periodontal situation and torque assessment for lower incisors before the Forsus FRD application. Some authors ${ }^{19,20}$ claimed that the gingival recession or bone loss could be detected if incisor proclination happened in class II patients. The gingival recession rate in the male is higher than that in the female, is higher in the mandible than that in the maxilla, and is higher on the facial surfaces than that on the lingual surfaces. ${ }^{21}$ And the final alveolar bone thickness rather than the initial alveolar bone thickness is significantly associated with the change in inclination. ${ }^{22}$ Prevention of unnecessary lower incisors proclination should be taken. Lace the entire lower brackets under the archwire with cinch back was used in the present study. The proclination of the mandibular incisors was still observed. More methods to increase lower incisors anchorage can be taken, such as greater size of the rectangular archwire, negative crown torque on the lower incisors, bracket with high negative torque, and miniscrew anchorage. Miniscrew anchorage 


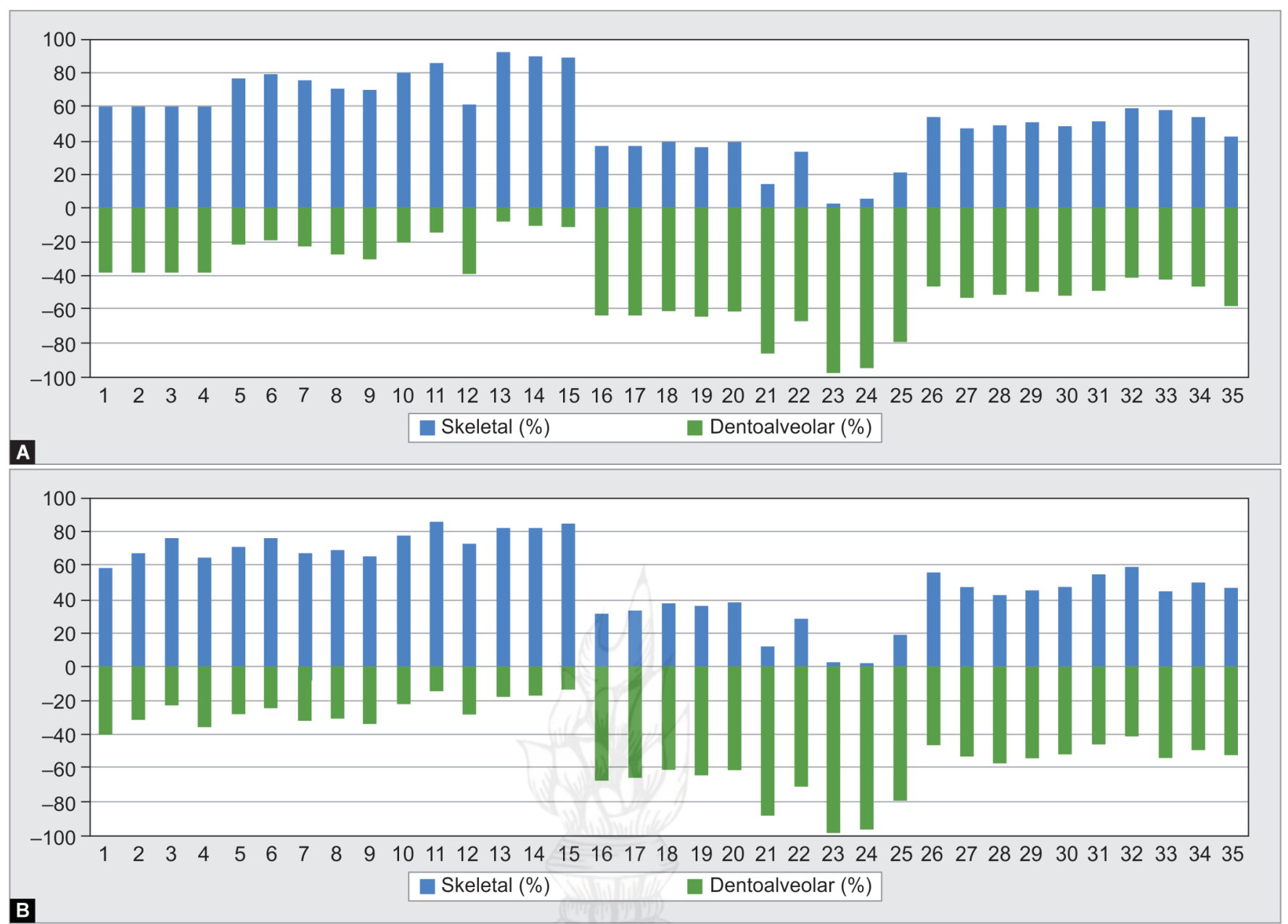

Figs $1 \mathrm{~A}$ and B: Fifteen patients with more than $60 \%$ skeletal contribution, 10 patients with more than $60 \%$ dentoalveolar contribution, 10 patients with almost equal skeletal and dentoalveolar contributions: (A) Overjet contributing; (B) Molar relation contributing

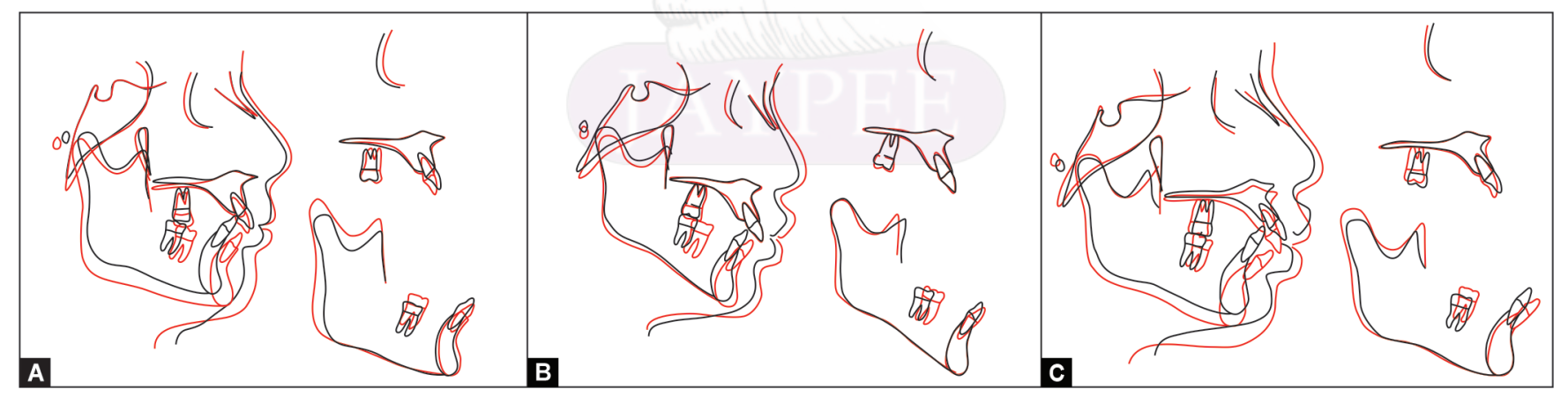

Figs 2A to C: Typical case: (A) Class II malocclusion corrected mainly by mandibular growth (SG); (B) Class II malocclusion corrected mainly by mandibular dentoalveolar mesial movement (DG); (C) Class II malocclusion corrected by both mandibular growth and mandibular dentoalveolar mesial movement (SDG)

has been focused by many authors to control the proclination of the mandibular incisors. ${ }^{23,24}$

\section{CONCLUSION}

The hypothesis was partially proved. Differential response to the Forsus FRD was observed in different cases. Skeletal and dentoalveolar effects played differentially in skeletal class II correction. Measures should be taken to prevent unnecessary lower incisors proclination in the Forsus FRD treatment. The mechanism of skeletal class II correction with the Forsus FRD may divide into mandibular growth, dentoalveolar effects, and both. Future studies should be carried out to investigate the mechanism of other functional appliance on the skeletal class II patient.

\section{Author Contribution}

Hegang Li and Xun Ren are authors contributed equally to this work. 


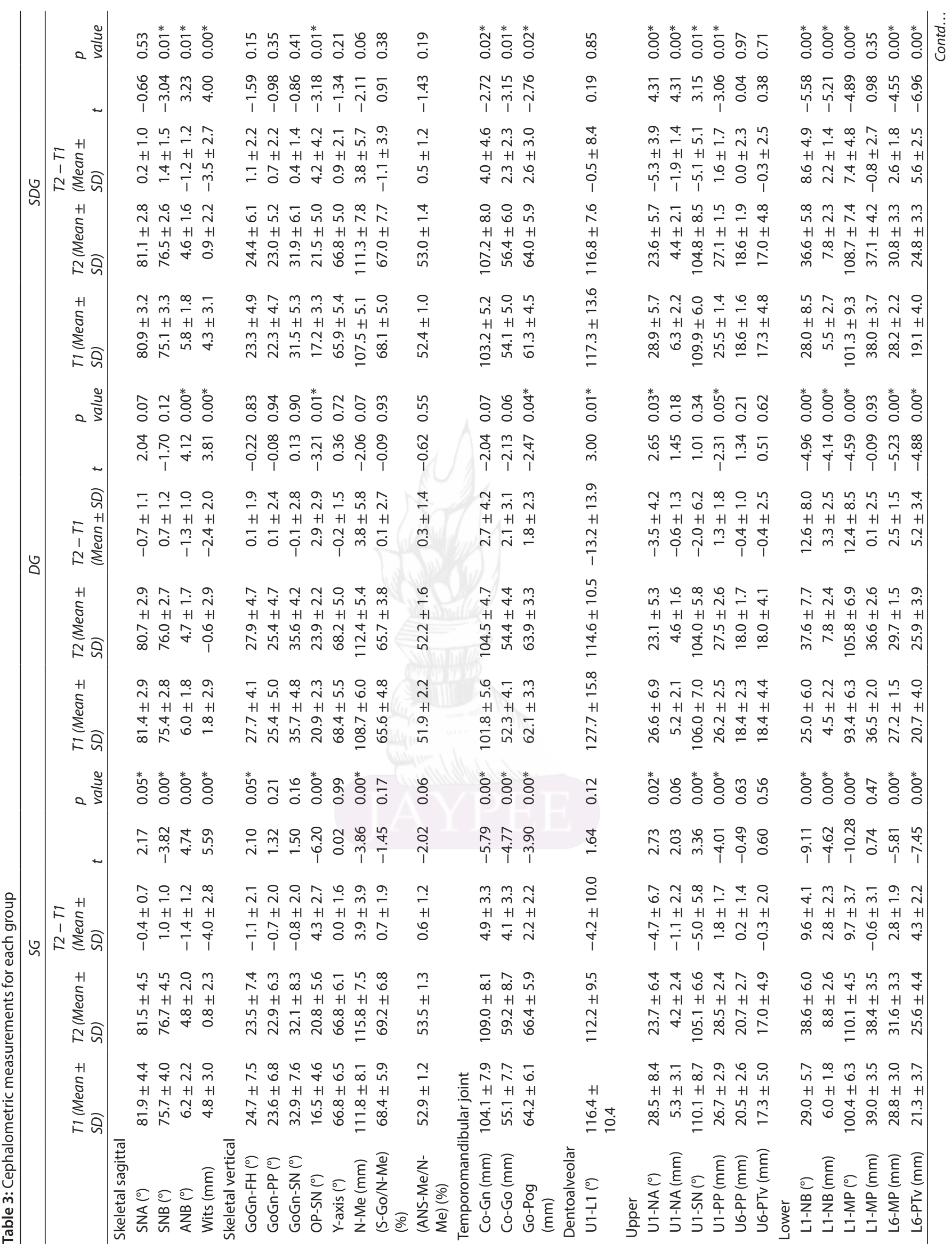




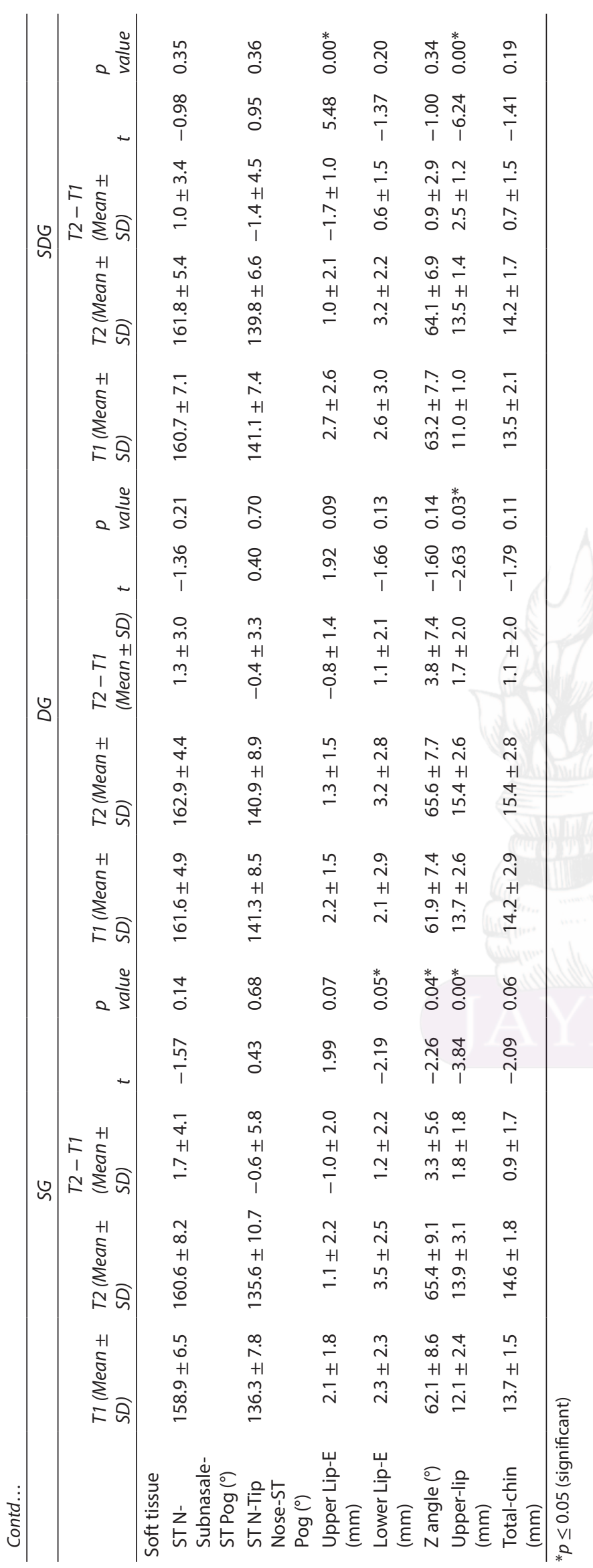

\section{References}

1. Baccetti T, Franchi L, Stahl F. Comparison of 2 comprehensive class II treatment protocols including the bonded Herbst and headgear appliances: a double-blind study of consecutively treated patients at puberty. Am J Orthod Dentofacial Orthop 2009;135(6):698 e1698 e-10. DOI: 10.1016/j.ajodo.2008.03.015; discussion-9.

2. Wahl N. Orthodontics in 3 millennia. Chapter 9: functional appliances to midcentury. Am J Orthod Dentofacial Orthop 2006;129(6):829-833. DOI: 10.1016/j.ajodo.2006.03.019.

3. Heinig N, Göz G. Clinical application and effects of the forsus spring. a study of a new Herbst hybrid. J Orofac Orthop 2001;62(6):436-450. DOI: 10.1007/s00056-001-0053-6.

4. El-Sheikh MM, Godfrey K, Manosudprasit M, et al. Force-deflection characteristics of the fatigue-resistant device spring: an in vitro study. World J Orthod 2007;8(1):30-36.

5. Franchi L, Alvetro L, Giuntini V, et al. Effectiveness of comprehensive fixed appliance treatment used with the forsus fatigue resistant device in class II patients. Angle Orthodontist 2011;81(4):678. DOI: 10.2319/102710-629.1.

6. Barnett GA, Higgins DW, Major PW, et al. Immediate skeletal and dentoalveolar effects of the crown- or banded type Herbst appliance on class II division 1 malocclusion. Angle Orthod 2008;78(2):361-369. DOI: 10.2319/031107-123.1.

7. Aras $A$, Ada E, Saracoğlu $H$, et al. Comparison of treatments with the forsus fatigue resistant device in relation to skeletal maturity: a cephalometric and magnetic resonance imaging study. Am J Orthod Dentofacial Orthop 2011;140(5):616-625. DOI: 10.1016/j. ajodo.2010.12.018.

8. Bisztriczky T, Fejes Tóth G. An improved Version of the cervical vertebral maturation (CVM) method for the assessment of mandibular growth. Angle Orthod 2002;72(4):316-323. DOI: 10.1043/0003-3219(2002)072<0316:AIVOTC>2.0.CO;2.

9. Pancherz $\mathrm{H}$. The mechanism of class II correction in Herbst appliance treatment. A cephalometric investigation. Am J Orthod 1982;82(2):104-113. DOI: 10.1016/0002-9416(82)90489-4.

10. Giuntini V, Vangelisti A, Masucci C, et al. Treatment effects produced by the twin-block appliance vs the forsus fatigue resistant device in growing class II patients. Angle Orthod 2015;85(5):784-789. DOI: 10.2319/090514-624.1.

11. Aras I, Pasaoglu A. Class II subdivision treatment with the forsus fatigue resistant device vs intermaxillary elastics. Angle Orthod 2017;87(3):371-376. DOI: 10.2319/070216-518.1.

12. Bock N, Pancherz $\mathrm{H}$. Herbst treatment of class II division 1 malocclusions in retrognathic and prognathic facial types. Angle Orthod 2006;76(6):930-941. DOI: 10.2319/100605-352.

13. Oztoprak MO, Nalbantgil D, Uyanlar A, et al. A cephalometric comparative study of class II correction with Sabbagh universal spring (SUS(2)) and forsus FRD appliances. Eur J Dent 2012;6(3):302-310. DOI: 10.1055/s-0039-1698965.

14. Stucki N, Ingervall B. The use of the jasper jumper for the correction of class II malocclusion in the young permanent dentition. Eur J Orthod 1998;20(3):271. DOI: 10.1093/ejo/20.3.271.

15. Männchen R. A critical evaluation of the pitchfork analysis. Eur J Orthod 2001;23(1):1-14. DOI: 10.1093/ejo/23.1.1.

16. Ruf S, Wüsten B, Pancherz H. Temporomandibular joint effects of activator treatment: a prospective longitudinal magnetic resonance imaging and clinical study. Angle Orthodontist 2002;72(6):527-540. DOI: 10.1043/0003-3219(2002)072<0527:TJEOAT>2.0.CO;2.

17. Gomes AS, Lima EM. Mandibular growth during adolescence. Angle Orthodontist 2006;76(5):786-790. DOI: 10.1043/0003-3219(2006)076[0786:MGDA]2.0.CO;2.

18. Karacay S, Akin EH, Gurton AU, et al. Forsus nitinol flat spring and jasper jumper corrections of class II division 1 malocclusions. Angle Orthodontist 2006;76(4):666-672. DOI: 10.1043/0003-3219(2006)076[0666:FNFSAJ]2.0.CO;2.

19. Coatoam GW, Behrents RG, Bissada NF. The width of keratinized gingiva during orthodontic treatment: its significance and impact on 
periodontal status. J Periodontol 1981;52(6):307-313. DOI: 10.1902/ jop.1981.52.6.307.

20. Artun J, Krogstad O. Periodontal status of mandibular incisors following excessive proclination. A study in adults with surgically treated mandibular prognathism. Am J Orthod Dentofacial Orthop 1987;91(3):225-232. DOI: 10.1016/0889-5406(87) 90450-1.

21. Nahas-Scocate AC, de Siqueira Brandao A, Patel MP, et al. Bone tissue amount related to upper incisors inclination. Angle Orthod 2014;84(2):279-285. DOI: 10.2319/031213-211.1.
22. Yodthong N, Charoemratrote C, Leethanakul C. Factors related to alveolar bone thickness during upper incisor retraction. Angle Orthod 2013;83(3):394-401. DOI: 10.2319/062912-534.1.

23. Aslan Bl, Kucukkaraca E, Turkoz C, et al. Treatment effects of the forsus fatigue resistant device used with miniscrew anchorage. Angle Orthod 2014;84(1):76-87. DOI: 10.2319/032613-240.1.

24. Eissa O, El-Shennawy M, Gaballah S, et al. Treatment outcomes of class II malocclusion cases treated with miniscrew-anchored Forsus fatigue resistant device: a randomized controlled trial. Angle Orthodontist 2017;87(6):824-833. DOI: 10.2319/032717-214.1. 